\title{
Searching for continuum $\gamma$-ray emission from OB associations with INTEGRAL, some preliminary results
}

\author{
J.-C. Leyder ${ }^{1,2}$ and G. Rauw ${ }^{1} \dagger$ \\ ${ }^{1}$ Institut d'Astrophysique et Géophysique, 17 Allée du 6-Août, 4000 Liège, Belgium \\ email: leyder@astro.ulg.ac.be \\ ${ }^{2}$ Integral Science Data Center, Versoix, Switzerland
}

\begin{abstract}
Recent studies suggested that there might be a correlation between unidentified $\gamma$-ray sources from the third EGRET catalogue and OB associations. Moreover, when extrapolating the fluxes measured by EGRET at energies above $100 \mathrm{MeV}$ with a power-law down to the energy range of ISGRI, the expected count rates should be large enough to be detectable with INTEGRAL. Most of those OB associations being located in the Galactic plane, they are monitored by INTEGRAL as part of the Core Program during both the Galactic Plane Scans and the Galactic Center Deep Exposure. Combining public and CP data, we have performed a search for gamma-ray emission from $\mathrm{OB}$ associations and the first results are presented.
\end{abstract}

Keywords. gamma rays: observations, X-rays: stars, open clusters and associations: general.

\section{OB Associations}

$\mathrm{OB}$ associations harbour massive $\mathrm{O}$ and B-type stars that exhibit fast stellar winds $\left(v_{\infty} \simeq 2000 \mathrm{~km} / \mathrm{s},\right)$ and large mass loss rates $\left(\dot{M} \simeq 10^{-5} M_{\odot} /\right.$ yr for O type stars $)$.

The presence of massives stars implies that these associations are found in star forming regions such as the spiral arms of the Galaxy.

\section{OB Associations in $\gamma$-rays}

Recent studies on the possible association of unidentified EGRET sources with different types of galactic objects indicated a significant correlation of sources from the $3 \mathrm{EG}$ catalogue with OB associations (Romero, Benaglia \& Torres 1999). Most of these OB associations are located in the Galactic Plane and are therefore observed during the INTEGRAL Core Programme. Deriving (accurate) positions and fluxes of these sources with the IBIS instrument might allow us to identify their counterparts. Furthermore, it might also help us determine whether the observed continuum emission is coming: (a) from radio-quiet pulsars (as suggested by Romero et al. 1999); (b) from shock fronts created by the interactions of the stellar winds of massive stars with the ambient ISM (Manchanda et al. 1996); (c) from hydrodynamic shocks in the winds of individual stars (Chen \& White 1991); or (d) from hydrodynamic shocks between the winds of massive binary systems (Eichler \& Usov 1993, Mücke \& Pohl 2001, Benaglia et al. 2001). The results of this study could thus help us to identify the so-far unknown counterparts of a large number of galactic $\gamma$-ray sources.

The sources listed by Romero et al. (1999) have photon indices near $\Gamma \sim 2$. Therefore the number of photons at a given energy is $n(E)=\alpha \times E^{-\Gamma}$. Using the EGRET fluxes

$\dagger$ Research Associate FNRS, Belgium. 


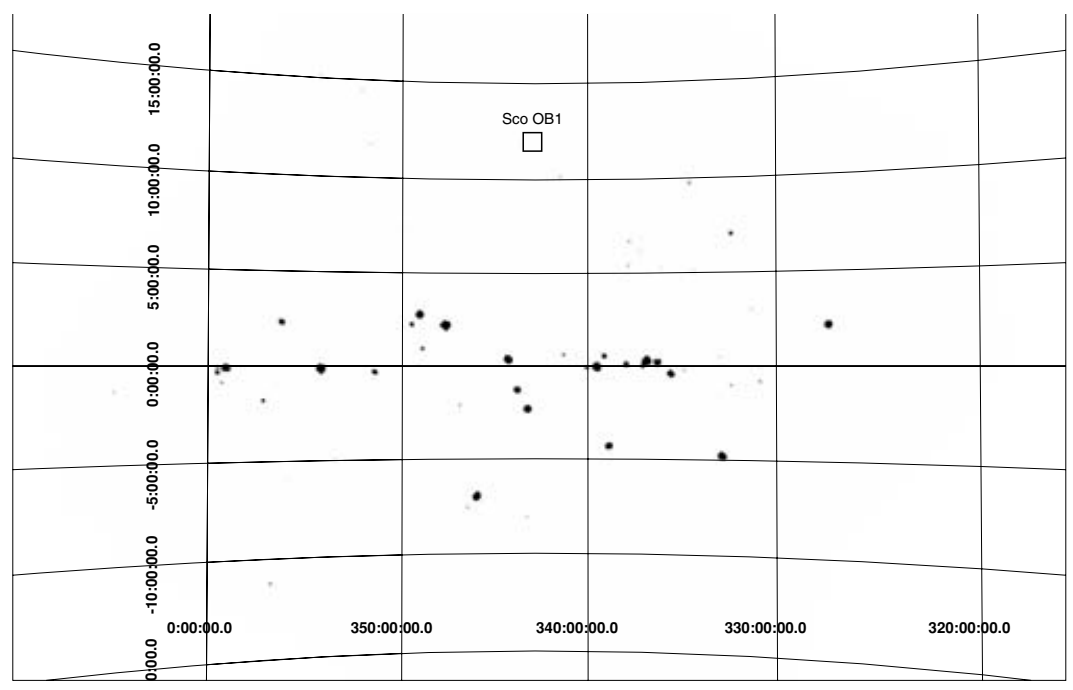

Figure 1. ISGRI significance map of the Sco OB 1 region in the $20-40 \mathrm{keV}$ band, produced with OSA 5.0. The fainter sources detected on this image have fluxes of $\sim 4$ mCrab.

( $>100 \mathrm{MeV}$ ) given by Romero et al. and extrapolating them with a power-law spectrum to the IBIS energy range $(15 \mathrm{keV}-10 \mathrm{MeV})$, we estimate for a typical OB association a continuum flux of $4510^{-9} \mathrm{ph} \mathrm{cm}^{-2} \mathrm{~s}^{-1} \mathrm{keV}^{-1} @ 1 \mathrm{MeV}$.

\section{Analysis with INTEGRAL}

Some preliminary results were first obtained with the Off-line Scientific Analysis (OSA) software, version 4.2. The analyses are being repeated with OSA 5.0 to take advantage of the better background reduction introduced with this new release.

The only instrument used up to now is ISGRI (Lebrun et al. 2003), a coded-mask imager offering a spatial resolution of $12^{\prime}$ over an energy range from approximately 23 to $1000 \mathrm{keV}$.

\section{Preliminary results}

The first tests carried out using OSA 4.2 did not permit to detect any signal for the sample of OB associations that were studied. The improvements in OSA 5.0, especially in the background correction, might help change this situation (see Figure 1).

\section{References}

Benaglia, P., Romero, G.E., Stevens, I.R., \& Torres, D. F. 2001, A\&A 366, 605

Chen, W. \& White, R.L. 1991, ApJ 266, 512

Eichler, D. \& Usov, V. 1993, ApJ 402, 271

Lebrun, F., Leray, J.P., Lavocat, P., et al. 2003, A\&SA 411, L141

Manchanda, R.K., Polcaro, V.F., Norci, L., Giovannelli, F., Brinkmann, W., Radecke, H.D., Manteiga, M., Persi, P., \& Rossi, C. 1996, A $ॄ$ A 305, 457

Mücke, A. \& Pohl, M. 2001, in: A.F.J. Moffat \& N. St.-Louis (eds.), Interacting Winds from Massive Stars, ASP Conf. Series, vol. 260, p. 355

Romero, G.E., Benaglia, P., \& Torres, D.F. 1999, A\&A 348, 868 